\title{
Un modelo ontológico espacial para el tratamiento de desórdenes de conducta infantil
}

\author{
María J. Somodevilla, Andrea M. P. Tamborrell, \\ Ivo H. Pineda, Concepción Pérez de Celis \\ Facultad de Ciencias de la Computación, \\ Benemérita Universidad Autónoma de Puebla, México

\begin{abstract}
Resumen. Los desórdenes de conducta son un comportamiento de patrones persistentes en niños y adolescentes en presencia de los cuales los derechos de otros o reglas básicas son violados. Considerando este problema, se propone la creación de un modelo ontológico para el tratamiento espacial de desórdenes de conducta en el idioma español. Este modelo ontológico considera la interacción de ontologías individuales, el cual permitirá responder a consultas espaciales basadas en semántica, tales como lugares de incidencia de cierto trastorno de conducta o interacciones medicamentosas en el tratamiento de cierto problema de conducta. En el diseño del modelo se aplican los conceptos de reusabilidad e integración de ontologías las cuales han sido individualmente axiomatizadas para lograr consistencia del sistema.
\end{abstract}

Palabras clave: Desórdenes de conducta, modelo ontológico espacial.

\section{An Ontological Model for Child Conduct Disorders}

\begin{abstract}
Conduct disorders are repetitive behavior patterns in child and adolescents where rules or basic rights of third parties are broken. Considering this problem, the creation of an ontological model for spatial treatment of conduct disorders in the Spanish language is proposed. This ontological model considers the interaction of individual axiomatized ontologies, which will be able to answer spatial queries based on semantic, such as incidence places of conduct disorder or drugs in the treatment of a behavioral problem. Concepts of ontologies' reusability and integration to achieve the system consistency along the model design have been incorporated.
\end{abstract}

Keywords. Conduct disorder, spatial ontological model. 


\section{Introducción}

Los desórdenes de conducta son una condición severa caracterizada por un comportamiento hostil y, en ocasiones violencia física. Infantes con problemas de conducta exhiben crueldad, que incluye desde maltrato físico a personas o animales hasta el acoso (bullying). Desde la infancia y adolescencia los problemas de conducta normalmente desarrollan en el adulto una personalidad antisocial, que necesita ser corregida con tratamiento tan pronto como sea detectada, si este problema es detectado a tiempo, mejor será el pronóstico [3]. Una ontología representa un modelo conceptual describiendo cierto dominio, tipos de objetos y conceptos que existen, así como sus propiedades y relaciones.

Considerando la gravedad de los desórdenes de conducta infantil, las tecnologías de la información podrían utilizarse como un soporte para su detección, así como una guía de orientación para padres. La Web se ha convertido en el principal medio de comunicación en nuestros días y son las ontologías las que proveen su base de conocimiento semántica. En este trabajo se propone desarrollar un sistema de ontologías que relaciona tres ontologías: desórdenes de conducta infantil, espacial de México y de psicofármacos.

\section{Ontologías biomédicas}

Existe trabajo previo en la FCC en el desarrollo de ontologías espaciales biomédicas a través de los proyectos en $[10,11,12]$. Dentro de estos proyectos se desarrollaron las ontologías GeoOntoMex y HealthOntoMex.

Diferentes ontologías se han creado en el ámbito médico como la Disease Ontology, la cual tiene como finalidad agrupar un vocabulario médico controlado desarrollado en el campo bioinformático, en colaboración con el Centro de Medicina Genética de la Universidad Northwestern, Chicago. Disease Ontology fue diseñada para facilitar una relación de las enfermedades y las condiciones asociadas a ellas para códigos médicos particulares como ICD9CM [1], SNOMED [2] y otros [9].

La ontología SNOMED [3] incluye una terminología de 364,000 conceptos de cuidado médico, con significados únicos y definiciones basadas en lógica formal organizadas en jerarquías [9]. DrOn [13] es una ontología para consultar fármacos por ingredientes, por disposición molecular y terapéutica, y por efectos secundarios.

La mayor parte de los trabajos ontológicos desarrollados en el ámbito de medicina están desarrollados en el idioma inglés y los pocos trabajos hechos de desórdenes de conducta no abordan específicamente este problema en infantes, por lo cual este trabajo representaría una gran diferencia en dicha área y en los países de habla hispana.

La publicación de ontologías médicas se puede realizar en sitios como biontology.org para que estas puedan ser de libre acceso en todo el mundo. Dicho sitio contiene una gran base de datos con ontologías del área de la medicina y permite a cualquier usuario subir su trabajo ontológico para que este pueda ser consultado por personas alrededor del mundo. Además, este sitio permite al usuario que lo visita ver a detalle 
los datos de la ontología tales como el nombre, el identificador único e incluso tiene un apartado para visualizar la ontología de manera gráfica en línea.

\section{Desórdenes de conducta}

Los desórdenes de conducta son una serie de problemas comportamentales y emocionales que se presentan en niños y adolescentes. Los problemas pueden involucrar comportamiento impulsivo o desafiante, consumo de drogas o actividad delictiva [7]. De acuerdo a [4], infantes con desorden de conducta pueden presentar cuatro tipos de comportamiento negativo como se detalla en 3.1 .

\subsection{Tipos de comportamiento}

- Agresión hacia personas o animales: (a) acoso y amenaza, (b) iniciar peleas físicas, (c) daño físico serio con el uso de armas, (d) asalto sexual.

- Destrucción de propiedad ajena: (a) daño intencional a la propiedad de otros, (b) iniciar incendios de manera deliberada.

- Acciones como engañar, mentir y robar: (a) allanamiento, (b) mentir para obtener cosas o favores o para evitar obligaciones, (c) robar sin confrontación.

- No respetar las reglas: (a) faltar a clases, (b) escapar de casa y/o pasar las noches sin permiso de los padres.

\subsection{Causas de los desórdenes de conducta}

Diferentes factores contribuyen a que una persona desarrolle desórdenes de conducta, incluyendo daño cerebral, abuso o negligencia, problemas genéticos, problemas escolares y experiencias traumáticas [4].

Existen diferentes tipos de factores de riesgo como:

- Biológicos: historial familiar de comportamiento negativo, desafiante o desorden de comportamiento.

- Modo de vida: violencia, divorcio, rechazo familiar.

\subsection{Tratamientos e intervenciones}

Está reportado en la bibliografía que los desórdenes de conducta se tratan con fisioterapia individual o grupal y/o con psicofármacos.

\subsubsection{Intervenciones psicosociales}

Sin intervención es probable que los desórdenes de comportamiento negativo (Disruptive Behavior Disorders) progresen [6]. Hay un número de tratamientos prometedores que están disponibles y que cuando son completados pueden llevar a grandes beneficios. Una investigación en [1] demostró que las personas que completan la 
"Terapia de Intervención Padre-Hijo" muestran un cambio significativo al terminar dicha terapia.

En [5] se han identificado 16 tratamientos basados en evidencias para los desórdenes de comportamiento. Dos ejemplos son:

- PMT [4] "Entrenamiento para padres". Está dirigido a padres y les enseña a identificar antecedentes, comportamientos resultantes y consecuencias asociadas a sus hijos así como también a ellos mismos. Por último, el entrenamiento se enfoca en reforzar conductas deseadas.

- PCIT [5] "Terapia de interacción padre-hijo". Enfatiza mejoras en la relación entre los padres e hijos y ofrece herramientas para ayudar a manejar comportamientos que son negativos.

\subsubsection{Psicofármacos}

Los psicofármacos se clasifican en ansiolíticos, antidepresivos, antimaníacos y antipsicóticos. Medicamentos antipsicóticos o neurolépticos son ampliamente utilizados en el tratamiento para el tratamiento de agresión aguda y crónica en varias poblaciones [14].

Estudios indican que antipsicóticos atípicos generalmente son más eficaces que un placebo en el tratamiento de agresiones, pero tienen diferentes efectos en cada persona. En menores de edad con problemas de desórdenes de comportamiento, la risperidona es el medicamento más estudiado.

\section{Propuesta de solución}

En esta sección se define el problema a resolver, se establece la metodología a utilizar en el desarrollo, así como los criterios de evaluación. Finalmente se llevan a cabo todas las etapas de desarrollo del sistema de ontologías propuesto.

\subsection{Definición del problema}

Los desórdenes de conducta infantil representan un problema de salud a nivel global, entonces las tecnologías de la información podrían utilizarse como un soporte para su detección, así como una guía de orientación para padres. En este sentido se realizará la creación de un sistema de ontologías reutilizable, usable y actualizable para tratar semánticamente los desórdenes de conducta en infantes así como sus tratamientos y las áreas geográficas de influencia.

\subsection{Metodología}

La metodología de diseño utilizada es una modificación de la propuesta por [2]. Los pasos de la metodología general para construcción de ontologías son: (1) Elicitación de términos, (2) Identificación de módulos, (3) Integración de módulos y (4) 
Evaluación global del sistema de ontologías, donde los módulos son las ontologías individuales.

Las características principales del sistema de ontologías son las siguientes:

- Diseño Orientado a Dominios. Dominio: Salud, subDominio: salud mental infantil.

- Ontología Orientada a la Reutilización: GeOntoMex [10], dominio: Geográfico. Esta ontología representa la división política administrativa de México.

- Ontología Modular: 3 ontologías individuales conectadas por axiomas semánticos.

- Ontología Incremental: Permite la definición de nuevos axiomas estructurales y de comportamiento.

La técnica que propone [2] para la extracción de términos que integrarán la ontología es la generación de preguntas de competencia relacionadas con el problema a resolver. Dichas preguntas se espera que el sistema de ontologías responda al final de su realización. Las siguientes son algunas de las preguntas de competencia:

1. ¿Cuáles son los síntomas del desorden de conducta "D”?

2. ¿Es "T" un tratamiento adecuado para la actitud "A"?

3. ¿Qué causas originan un desorden de conducta " $\mathrm{D}$ ”?

4. ¿Cuáles son los tratamientos indicados para un desorden de conducta "D"?

5. ¿En qué área geográfica han sido reportados casos del desorden de conduc-ta “D”?

\subsection{Evaluación de la ontología}

La consistencia de la ontología es evaluada por el razonador a través de los axiomas que se definen para establecer: las relaciones jerárquicas (taxonómicas), (2) las relaciones de tipo: data properties, es decir, preguntarse ¿cuáles características son suficientes y necesarias para definir a un concepto? y (3) las relaciones de tipo object properties, es decir, ¿se han cubierto todas las relaciones explícitas de las preguntas de competencia?

Por otra parte, se utilizan tres métodos de evaluación para la ontología, los cuales consisten en: (1) evaluación del conocimiento y conceptualización, es decir, el grado de cumplimiento del listado de las preguntas de competencia previamente establecidas, (2) evaluación de la calidad, grado de cumplimiento con los principios de diseño y (3) evaluación de rendimiento, velocidad de las respuestas que el razonador generará.

\subsection{Diseño conceptual de ontologías individuales}

El sistema se compone de tres ontologías individuales. Las ontologías de desórdenes de conducta y la de psicofármacos fueron expresamente creadas para este proyecto y la ontología espacial fue adaptada y reutilizada. 


\subsubsection{Ontología de desórdenes de conducta}

La ontología de desórdenes de conducta consta de diferentes clases, individuos, propiedades de individuos y propiedades de objeto (relaciones entre clases) como se muestra en la Figura 1. Las clases son: Causa, Desorden de conducta, Paciente, Síntoma y Tratamiento.

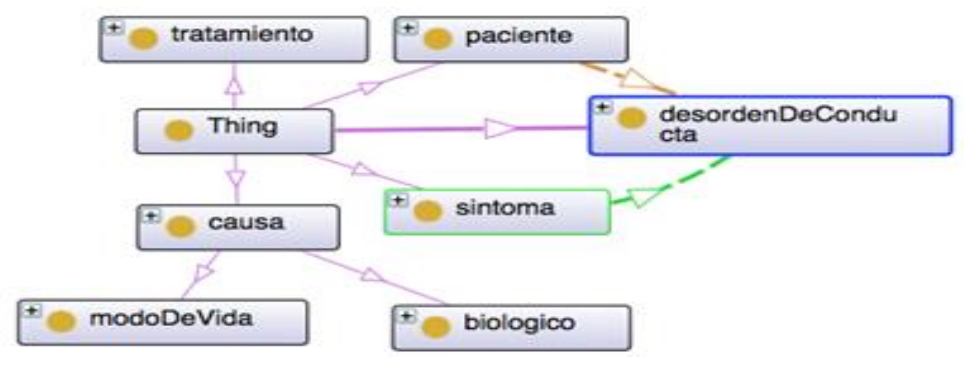

Fig. 1. Ontología OntoDesorden.

Las relaciones entre clases se presentan en la Tabla 1.

Tabla 1. Relaciones entre clases.

\begin{tabular}{lll}
\hline Relación & Dominio & Rango \\
\hline tieneCausas & Desorden de conducta & Causa \\
tieneSintomas & Desorden de conducta & Síntoma \\
tieneTratamiento & Desorden de conducta & Tratamiento \\
recomiendaTratamiento & Paciente & Tratamiento \\
tieneDesorden & Paciente & Desorden de conducta \\
\hline
\end{tabular}

Los individuos de las clases tienen las propiedades que se presentan en la Tabla 2.

Tabla 2. Propiedades de las clases.

\begin{tabular}{|c|c|}
\hline Clase & Dominio \\
\hline Causa & $\begin{array}{l}\text { causaNombre } \\
\text { causaTipo } \\
\text { causaDescripcion }\end{array}$ \\
\hline Desorden de conducta & $\begin{array}{l}\text { DesordenNombre } \\
\text { desordenTipo }\end{array}$ \\
\hline Paciente & $\begin{array}{l}\text { pacienteEdad } \\
\text { pacienteGenero } \\
\text { pacienteID }\end{array}$ \\
\hline Síntoma & $\begin{array}{l}\text { sintomaNombre } \\
\text { sintomaIntensidad } \\
\text { sintomaDescripcion }\end{array}$ \\
\hline Tratamiento & $\begin{array}{l}\text { tratamientoNombre } \\
\text { tratamientoTipo } \\
\text { tratamientoDuración }\end{array}$ \\
\hline
\end{tabular}




\subsubsection{Ontología de psicofármacos}

La ontología de psicofármacos se muestra en la Figura 2. Las clases son: Ansiolítico, Antidepresivo y Antipsicótico.

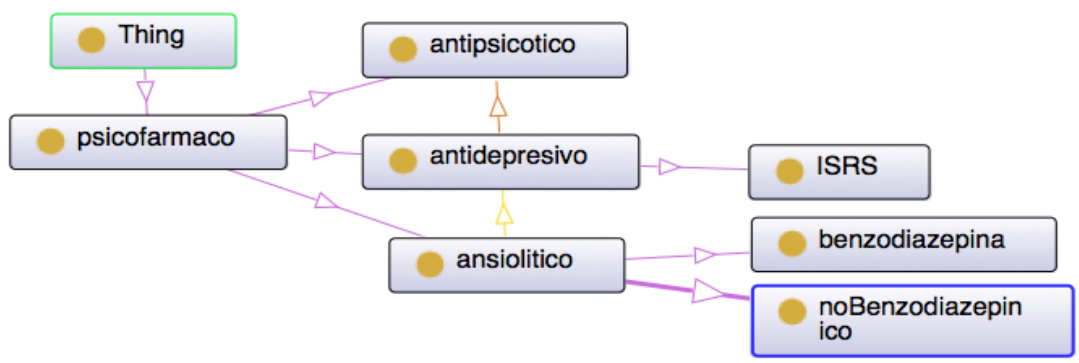

Fig. 2. Ontología Psicofármaco.

Las propiedades entre clases de la ontología psicofármacos se describen en la Tabla 3.

Tabla 3. Propiedades de las clases.

\begin{tabular}{ll}
\hline Clase & Dominio \\
\hline Psicofarmaco & $\begin{array}{l}\text { farmacoNombre } \\
\text { farmacoOtroNombre } \\
\text { farmacoDescripcion } \\
\text { antipsicoticoGenerico } \\
\text { antipsicoticoGeneracion }\end{array}$ \\
Antipsicotico & ISRSGenerico \\
& noBenzoGenerico \\
ISRS & benzoAccion \\
noBenzodiazepinico & benzoGenerico \\
benzodiazepina & \\
\hline
\end{tabular}

Las relaciones entre clases se muestran en la Tabla 4.

Tabla 4. Relaciones entre clases.

\begin{tabular}{lll}
\hline Relación & Dominio & Rango \\
\hline interaccionMedAns & Ansiolitico & Antidepresivo \\
interaccionMedDep & Antidepresivo & Antipsicotico \\
\hline
\end{tabular}

\subsubsection{GeOntoMex}

Esta ontología es reutilizada porque es importante dar respuesta a consultas semánticas tales como la pregunta de competencia 5.

En la figura 3 se muestra el diseño conceptual de la ontología. Para el sistema se utiliza la clase de ExtensionGeopolitica que contiene la definición taxonómica política administrativa de México. 


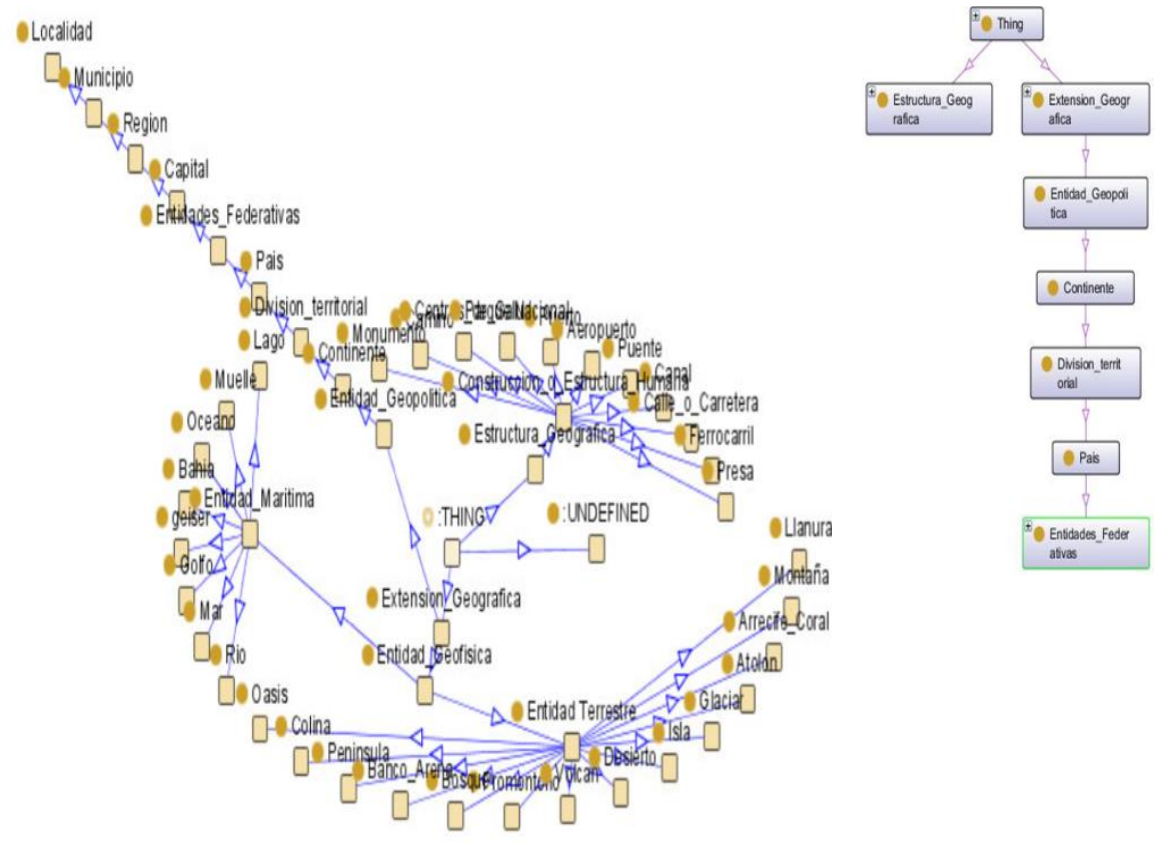

Fig. 3. (a) GeOntoMex.

(b) Reutilización de GeOntoMex.

\subsection{Creación del sistema de ontologías}

El sistema de ontologías representa la interacción de las ontologías individuales a través de las relaciones entre éstas (object properties). Esta forma de relación garantiza un modelo de diseño modular, interactivo e iterativo es decir se mantienen aparte los individuos de cada entidad lógica y al mismo tiempo pueden cooperar con los individuos de otras ontologías para generar nuevo conocimiento. La figura 4 muestra el esquema contextual del sistema de ontologías propuesto. Se muestran las tres ontologías y las relaciones Psicofarmaco sePrescribepara Desorden y Desorden seLocalizaen Mexico.

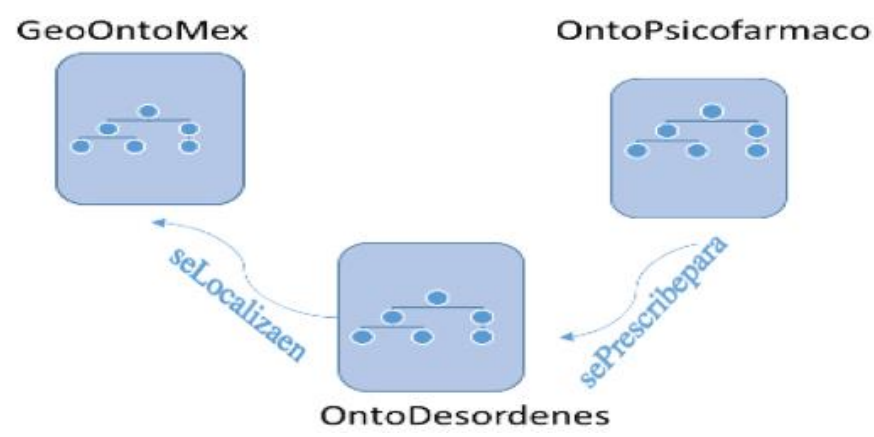

Fig. 4. Sistema de ontologías propuesto. 


\subsection{Consultas en DL Query}

Las reglas DL (Description Logic) se definieron para responder una selección de las preguntas de competencia enlistadas en la sección 4.2. En la Tabla 5 se muestran dichas preguntas de competencia, así como la regla que le da respuesta.

Tabla 5. Definición de DL queries.

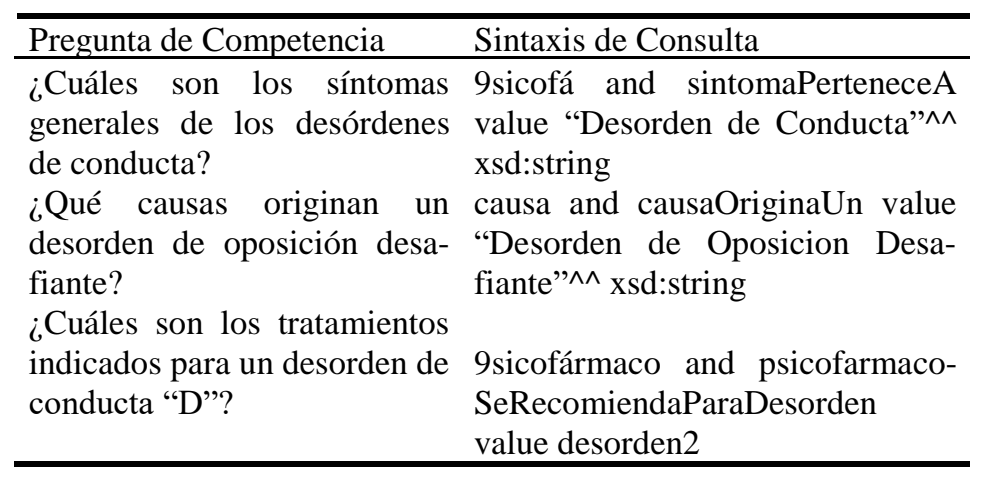

En la sección 5 se presentan los resultados de la clasificación reportados por Prótégé 5.0.0.

\section{Resultados}

$\mathrm{Al}$ ejecutar un razonador sobre la ontología, esta no registra ningún error por lo cual se concluye que la ontología es consistente.

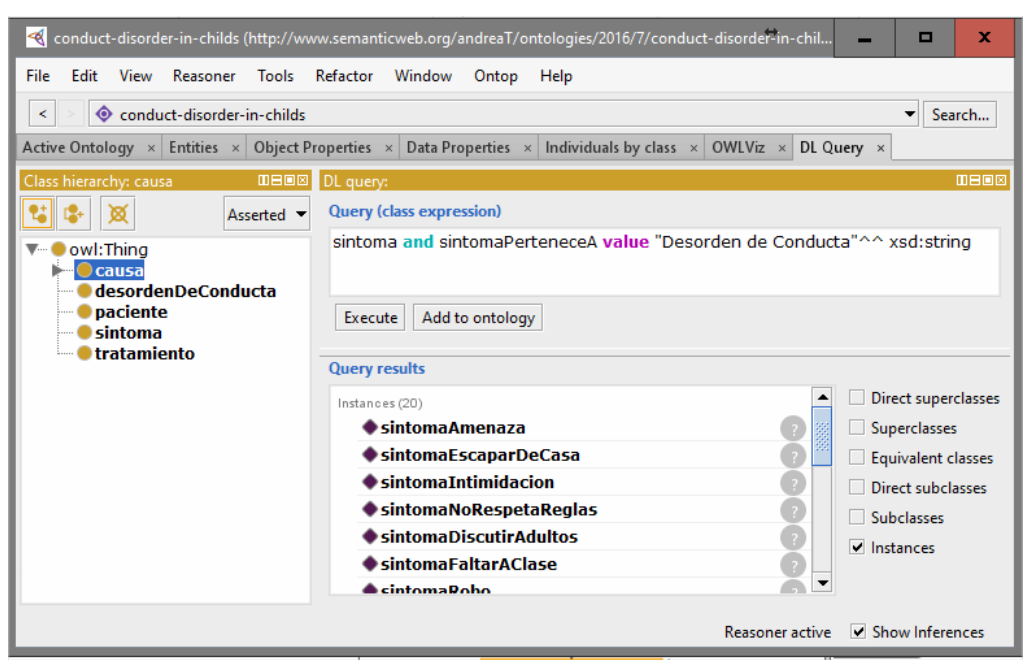

Fig. 5. Ejecución de primera pregunta de competencia. 


\subsection{Ejecución de DL query}

En las figuras 5, 6 y 7 se muestra la ejecución las preguntas de competencia descritas en la tabla 5.

En la Figura 5 se muestran todos los síntomas que han sido capturados como individuos de la clase síntoma y que se asocian a los desórdenes de conducta infantil.

En la Figura 6 se muestran los resultados de la ejecución de la expresión de clase que determina las causas que originan un desorden de oposición desafiante.

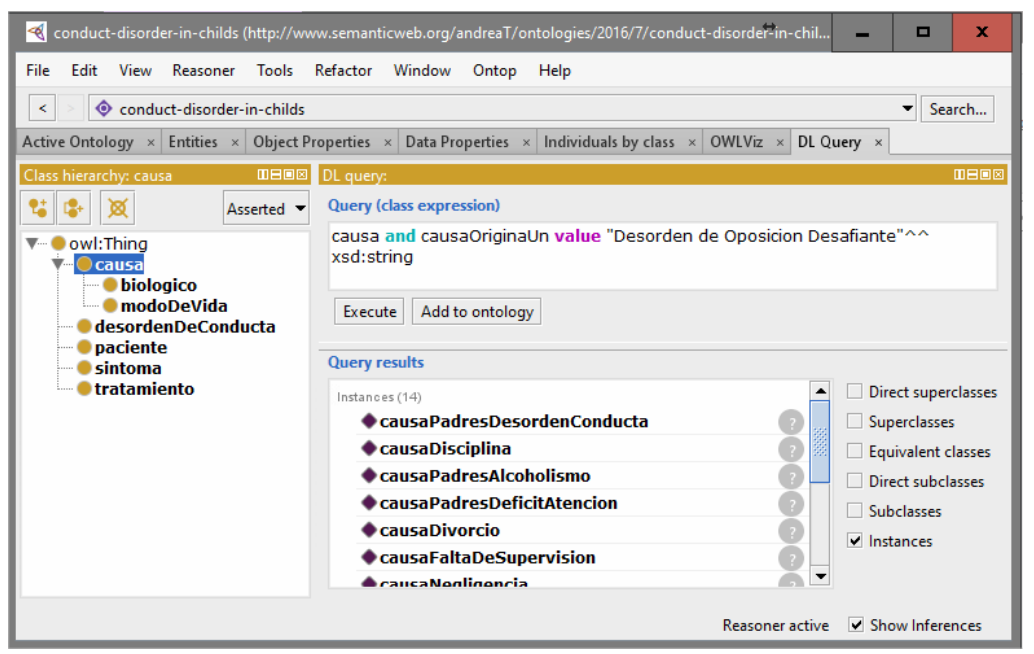

Fig. 6. Ejecución de segunda pregunta de competencia.

La Figura 7 presenta los resultados de la consulta asociada con el tratamiento con psicofármacos indicados para el desorden2. Los individuos por el momento son genéricos pues se está comprobando está información con los expertos.

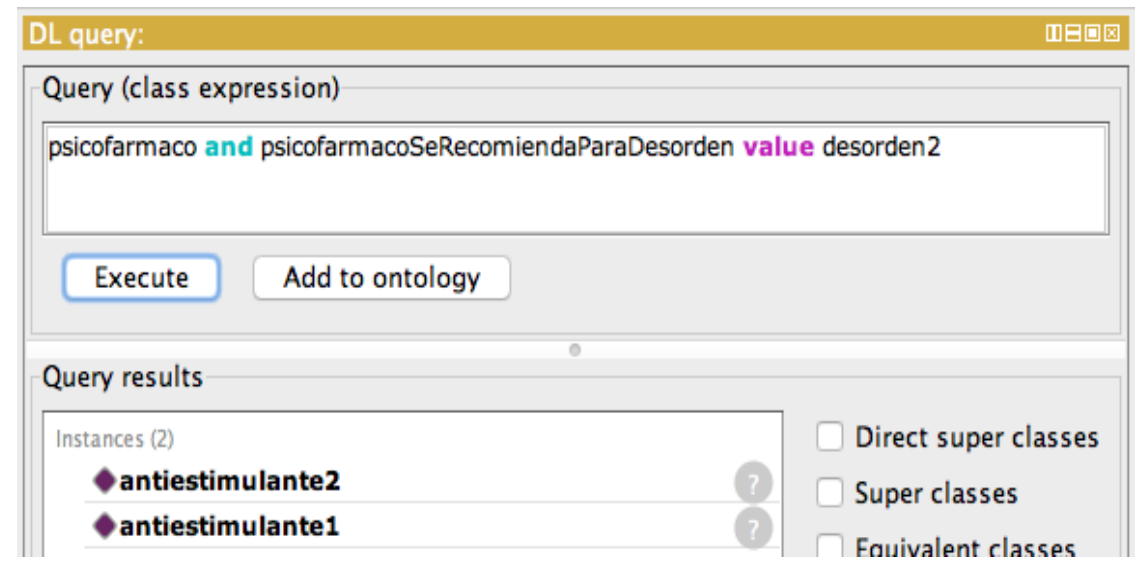

Fig. 7. Ejecución de tercera pregunta de competencia. 


\section{Conclusiones}

Las alteraciones de la conducta en la infancia, han pasado a ser no solo el motivo de consulta más frecuente en psiquiatría infanto-juvenil, sino un motivo de alarma para la sociedad en general. Se propuso un sistema de ontologías que integra a través propiedades de los objetos tres ontologías individuales: desórdenes de conducta, geográfica de México y de psicofármacos. Se implementan además las relaciones entre clases que posteriormente se usaron en la definición de reglas DL para dar respuesta a las preguntas de competencia. El sistema de ontologías propuesto es consistente de acuerdo con la validación realizada por Protégé 5.0.0, donde fueron validados los axiomas de clase, de relaciones y de individuos.

Como trabajo a futuro se está trabajando en la definición una metodología, para el poblado semiautomático del sistema de ontologías propuesto, soportado por conceptos y herramientas de PLN e IR. Esta metodología comprende desde la búsqueda de casos clínicos, síntomas y tratamientos en diferentes sitios Web de la especialidad hasta la inserción de individuos. La población semiautomática permitirá el rápido crecimiento de individuos en la base de conocimientos para lograr una mejor precisión y orientación en los casos de desórdenes de conducta.

La presente propuesta tendrá un impacto social puesto que se plantea que pueda ser utilizado como base de conocimiento, en un sistema para la toma de decisiones relacionado con desórdenes de conducta infantil en México.

\section{Referencias}

1. Boggs, S.R., Eyberg, S.M., Edwards, D.L., Rayfield, A., Jacobs, J., Bagner, D., Hood, K.K.: Outcomes of parent-child interaction therapy: A comparison of treatment completers and study dropouts one to three years later. Child \& Family Behavior Therapy (2004)

2. Bravo, M., Perez, J., Velazquez, J., Sosa, V., Montes, A., Lopez, M.: Design of a shared ontology used for translating negotiation primitives. International Journal of Web and Grid Services, 237-259 (2006)

3. Child Mind Institute. Guide to conduct Disorder, What is it?

4. Desórdenes de la Conducta. American Academy of Child Adolescent Psychiatry (2004)

5. Eyberg, S.M., Nelson, M.M., Boggs, S.R.: Evidence-based psychosocial treatments for children and adolescents with disruptive behavior (2008)

6. Gathright, M., Tyler, L.: Disruptive Behaviors in Children and Adolescents (2012)

7. Medline Plus.: Trastorno de Conducta Biblioteca Nacional de Medicina de los EEUU (2015)

8. National Institutes of Health. SNOMED CT. U.S. National Library of Medicine (2016)

9. Sarrión, A., Corrales, Y.: Un acercamiento a las ontologías médicas y su importancia. IV Jornada Científica de la SOCECS

10. Somodevilla, M.: Desarrollo de Sistemas de Ontologías para descubrir patrones de estilo de vida asociados con enfermedades crónicas no transmisibles. SOGM-ING15-I VIEP (2015)

11. Somodevilla, M.: Desarrollo de técnicas para el procesamiento de consultas con datos espaciales biomédicos. SOGM-ING14-I VIEP (2014)

12. Somodevilla, M.: Desarrollo de Técnicas para Poblado Automático de Ontologías Espaciales Biomédicas. SOGM-ING15-I VIEP (2016) 
María J. Somodevilla, Andrea M. P. Tamborrell, Ivo H. Pineda, Concepción Pérez de Celis

13. Werry, J.S., Aman, M.G.: Methylphenidate and haloperidol in children: Effects on attention, memory and activity (1975)

14. Duncan, J., Eilbeck, K., Narus, S.P., Clyde, S., Thornton, S., Staes, C.: Building an Ontology for Identity Resolution in Healthcare and Public Health. Online journal of public health informatics 7 (2) (2015)

15. He, Y., Sarntivijai, S., Lin, Y., Xiang, Z., Guo, A., Zhang, S., Smith, B.: OAE: The ontology of adverse events. Journal of biomedical semantics, 5(1) (2014) 